\title{
Entire solutions of the Fisher-KPP equation in time periodic media
}

\author{
Wei-Jie Sheng and Mei-Ling Cao \\ Communicated by $Y$. Charles Li, received September 11, 2011.
}

\begin{abstract}
This paper is concerned with the existence of the pulsating type entire solutions of the Fisher-KPP equation with advection term in time periodic media. By constructing appropriate subsolutions and supersolutions, we prove that there exists a pulsating type entire solution which behaves as two pulsating traveling fronts coming from two opposite directions and approaching each other. The main technique here is to characterize the asymptotic behavior of the pulsating traveling front as $t \rightarrow-\infty$.
\end{abstract}

\section{CONTEnTs}

1. Introduction 133

2. Preliminaries 137

3. Existence of the Pulsating Entire Solution 139

4. Discussion 143

5. Acknowledgements 143

References 143

\section{Introduction}

In the present paper, we consider the nonlinear evolutionary equation in time periodic media of the following form:

(1.1)

$\left\{\begin{array}{l}u_{t}-\nabla \cdot(A(t, y) \nabla u(t, x, y))+q(t, y) \cdot \nabla u(t, x, y)=f(t, y, u) \quad \text { in }(t, x, y) \in \mathbb{R} \times \bar{\Omega}, \\ \nu A \nabla u=0 \quad \text { on }(t, x, y) \in \mathbb{R} \times \partial \Omega,\end{array}\right.$

1991 Mathematics Subject Classification. 35

Key words and phrases. Reaction-advection-diffusion equations, Time periodic media, Entire solutions.

Wei-Jie Sheng is supported by NSF of China (No.11031003).

(c)2012 International Press 
where $\Omega=\left\{(x, y) \in \mathbb{R}^{d} \times \omega\right\}$ is a smooth unbounded domain and $\omega$ is a $C^{2, \alpha}$ bounded domain of $\mathbb{R}^{\mathcal{N}-d}$.

Equation (1.1) is the generalization of the homogeneous reaction-diffusion equation

$$
u_{t}-\triangle u=f(u) \text { in } \mathbb{R}^{\mathcal{N}} .
$$

For the homogeneous equation (1.2), the most interesting problem is the so called planar traveling front and its qualitative properties, which have first been investigated by Fisher $[\mathbf{9}]$ and Kolmogorov, Petrovsky and Piskunov [19] in their pioneering articles. Here a planar traveling front is a solution of the form $u(t, x)=$ $U(x \cdot e+c t)$ with $e$ a unit vector and $c$ the propagation speed in the direction $e$. For more detailed results, we refer to Chen [6], Hamel and Nadirashvili [16] and Volpert et al. [38].

In this paper we only concerned with pulsating traveling fronts. For the equation (1.1), when the variable $t$ in $A, q$ and $f$ is replaced by the variable $x$ and are $L_{i}$ periodic with respect to the variable $x_{i}$, Freidlin and Gartner [13] and Freidlin [12] investigated propagation phenomena by using a stochastic method. However, they did not give the proof of the existence of fronts. Xin [44] first proved existence of pulsating traveling waves in the form $u=\phi(x \cdot e+c t, x)$. Shigesada, Kawasaki and Teramoto $[\mathbf{3 5}, \mathbf{3 6}]$ first defined the notion of pulsating traveling fronts, which is a generalization of the notion of planar fronts to space periodic environments. Namely, a classical solution of equation (1.1) that satisfies:

$$
\left\{\begin{array}{l}
\forall x \in \mathbb{R}^{d}, t \in R, u(t+L \cdot e / c, x, y)=u(t, x+L, y), \\
u(t, x, y) \rightarrow 0 \text { as } x \cdot e \rightarrow-\infty \text { and } u(t, x, y) \rightarrow 1 \text { as } x \cdot e \rightarrow+\infty,
\end{array}\right.
$$

where $L=\left(L_{1}, \ldots, L_{d}\right)$ and each $L_{i}$ are positive numbers, $c$ is the wave speed, 0 and 1 are the only zeros of the reaction term $f(x, y, \cdot)$ for all $(x, y) \in \mathbb{R}^{\mathcal{N}}$. However, they only carried out numerical approximations and heuristic computations. One can easily check that a pulsating traveling front is a solution of the form $u(t, x, y)=$ $\phi(x \cdot e+c t, x, y)$, where $\phi$ is periodic in $x$ and satisfies

$$
\lim _{z \rightarrow-\infty} \phi(z, x, y) \rightarrow 0, \quad \lim _{z \rightarrow+\infty} \phi(z, x, y) \rightarrow 1, \quad z=x \cdot . e+c t
$$

For a fully space periodic environment with positive nonlinearity, Berestycki and Nirenbeg [2] and Berestycki et al. [3] gave the analytical result by using the same definition as Shigesada, Kawasaki and Teramoto $[\mathbf{3 5}, \mathbf{3 6}]$. Then Fréjacques [10] extended the notion of the pulsating traveling front to the time periodic environment with positive nonlinearity. Namely, a solution $u$ that satisfies

$$
\left\{\begin{array}{l}
\forall x \in \mathbb{R}^{d}, t \in R, u(t+T, x, y)=u(t, x+c T e, y), \\
u(t, x, y) \rightarrow 0 \text { as } x \cdot e \rightarrow-\infty \text { and } u(t, x, y) \rightarrow 1 \text { as } x \cdot e \rightarrow+\infty,
\end{array}\right.
$$

where $T$ is the period. The pulsating traveling front for the bistable nonlinearity was studied by Alikakos et al. [1]. Recently, Nolen and Xin [34] studied traveling waves in space-time periodic shear flows, in the form $u=\phi(x \cdot e+c t, y, t)$. Nadin [31] extended the results to the space-time periodic media in terms of the contribution of Nolen et al. $[\mathbf{3 2}, \mathbf{3 3}]$. For the results of generalized pulsating traveling fronts, we refer to Berestycki and Hamel [4].

Next we give some assumptions on the coefficients and the nonlinearity. For the diffusion matrix $A(t, y)=\left(A_{i, j}(t, y)\right)_{1 \leq i, j \leq N}$, we assume that it is of class 
$C_{t ; y}^{1, \alpha / 2 ; 2, \alpha}(\mathbb{R} \times \bar{\omega})$ satisfying

$$
\Sigma_{1 \leq i, j \leq N} A_{i j}(t, y) \xi_{i} \xi_{j} \geq \alpha_{0}|\xi|^{2}, \quad \forall(t, y) \in \mathbb{R} \times \bar{\omega}, \quad \xi_{i}, \xi_{j} \in \mathbb{R}^{\mathcal{N}}, 1 \leq i, j \leq N,
$$

where $\alpha_{0} \geq 0$ is some constant. The advection term $q(t, y)=\left(q_{i}(t, y)\right)_{1 \leqslant i \leqslant N}$ is of class $C_{t ; y}^{1, \alpha / 2 ; 2, \alpha}(\mathbb{R} \times \bar{\omega})$. Assume that equation (1.1) has two stationary solutions $p^{ \pm}(t, y)$, where $p^{ \pm}(t, y) \in C_{t ; y}^{1, \alpha / 2 ; 2, \alpha}(\mathbb{R} \times \bar{\omega})$ satisfies

$\left\{\begin{array}{l}p_{t}^{ \pm}-\nabla \cdot\left(A(t, y) \nabla p^{ \pm}(t, y)\right)+q(t, y) \cdot \nabla p^{ \pm}(t, y)=f(t, y, u) \quad \text { in }(t, x, y) \in \mathbb{R} \times \bar{\Omega} \\ \nu A \nabla p^{ \pm}=0 \quad \text { on }(t, x, y) \in \mathbb{R} \times \partial \Omega .\end{array}\right.$

Without loss of generality, we assume that $p^{-}(t, y) \leq p^{+}(t, y)$ are periodic in $t$. Note that, by doing an easy translation, we always assume that equation (1.1) has two equilibrium: 0 and $p(t, y) \geq 0$ in the sequel.

We assume that the nonlinearity $f(t, y, u)$ is of class $C_{t ; y}^{1, \alpha / 2 ; 2, \alpha}(\mathbb{R} \times \bar{\omega})$ with respect to $(t, y)$ locally uniformly in $u \in \mathbb{R}$ and $\partial f / \partial u$ exists and is continuous in $\mathbb{R} \times \bar{\omega} \times \mathbb{R}$ and satisfies the following monostable condition:

$$
\left\{\begin{array}{l}
f(t, y, 0)=0, \quad \frac{\partial f}{\partial s}(t, y, 0)>0 \text { for all }(t, y) \in \mathbb{R} \times \bar{\omega} \\
f(t, y, s)>0, \quad \forall s \in(0, p(t, y)) \quad \text { for some }(t, y) \in \mathbb{R} \times \bar{\omega}, \\
\text { for all }(t, y) \in \mathbb{R} \times \bar{\omega}, \text { and for some } \sigma>0,0 \leq s \leq s^{\prime} \leq \sigma, \text { we have } \\
f(t, y, p(t, y)-s) \leq f\left(t, y, p(t, y)-s^{\prime}\right) .
\end{array}\right.
$$

All functions $A_{i j}, q_{i}$ and $f(\cdot, \cdot, u$ ) (for all $u \in \mathbb{R}$ ) are assumed to be periodic with respect to $t$, Namely,

$$
\begin{array}{ll}
A_{i j}(t+T, y)=A_{i j}(t, y), & q_{i}(t+T, y)=q_{i}(t, y), \\
f(t+T, y, u)=f(t, y, u), & (t, y) \in \mathbb{R} \times \bar{\omega},
\end{array}
$$

where $T>0$ is a fixed constant.

Define

$$
\zeta(t, y):=\frac{\partial f}{\partial u}\left(t, y, p^{-}(t, y)\right)
$$

which belongs to $C_{t ; y}^{1, \alpha / 2 ; 1, \alpha}(\mathbb{R} \times \bar{\omega})$. Assume that there is $\rho$ such that $0<\rho<$ $\min _{\mathbb{R} \times \bar{\omega}} p(t, y)$ and, for any classical bounded supersolution $\hat{u}$ of (1.1) satisfying $\hat{u}<p(t, y)$ and $\Omega_{\hat{u}}=\{\hat{u}(t, x, y)>p(t, y)-\rho\} \neq \emptyset$, there exists a family of functions $\left(\rho_{\tau}\right)_{\tau \in[0,1]}$ defined in $\bar{\Omega}_{\hat{u}}$ such that

$$
\left\{\begin{array}{l}
\tau \mapsto \rho_{\tau} \text { is continuous in } C_{t ;(x, y)}^{1+\alpha / 2 ; 2+\alpha}\left(\bar{\Omega}_{\hat{u}}\right), \\
\tau \mapsto \rho_{\tau}(t, x, y) \text { is nondecreasing for each }(t, x, y) \in \bar{\Omega}_{\hat{u}}, \\
\rho_{0}=0, \rho_{1} \geq \rho, \inf _{\bar{\Omega}_{\hat{u}}} \rho_{\tau}>0 \text { for each } \tau \in(0,1], \\
\left(\hat{u}+\rho_{\tau}\right)_{t}-\nabla \cdot\left(A \nabla\left(\hat{u}+\rho_{\tau}\right)\right)+q \cdot \nabla\left(\hat{u}+\rho_{\tau}\right) \geq f\left(x, y, \hat{u}+\rho_{\tau}\right) \quad \text { in } \bar{\Omega}_{\hat{u}}, \\
\nu A \nabla\left(\hat{u}+\rho_{\tau}\right) \geq 0 \quad \text { on }(\mathbb{R} \times \partial \Omega) \cap \Omega_{\hat{u}, \tau},
\end{array}\right.
$$

where $\Omega_{\hat{u}, \tau}=\left\{(t, x, y) \in \Omega_{\hat{u}}, \hat{u}(t, x, y)+\rho_{\tau}(t, x, y)<p(t, y)\right\}$. We shall also assume that there are $\beta>0$ and $\gamma>0$ such that the map

$$
(t, y, s) \mapsto \frac{\partial f}{\partial u}(t, y, s) \text { belongs to } C^{0, \beta}(\mathbb{R} \times \bar{\omega} \times[0, \gamma]),
$$


and

$$
f(t, y, s) \leq \zeta(t, y) s, \quad(t, y) \in \mathbb{R} \times \bar{\omega}, s \in[0, p(t, y)] .
$$

Given a unit vector $e \in \mathbb{R}^{d} \times\{0\}^{\mathcal{N}-d}$, a pulsating traveling front $u(t, x, y)$ of (1.1) connecting 0 and $p(t, y)$ travels in the direction $e$ with mean speed $c \in \mathbb{R}^{*}$ satisfying

$$
\left\{\begin{array}{l}
u_{t}-\nabla \cdot(A(t, y) \nabla u(t, x, y))+q(t, y) \cdot \nabla u(t, x, y)=f(t, y, u) \quad \text { in }(t, x, y) \in \mathbb{R} \times \bar{\Omega}, \\
\nu A \nabla u=0 \quad \text { on }(t, x, y) \in \mathbb{R} \times \partial \Omega \\
0<u(t, x, y)<p(t, y) \quad \text { for all }(t, x, y) \in \mathbb{R} \times \bar{\Omega} .
\end{array}\right.
$$

Let $u(t, x, y):=\phi(t, c t-x \cdot e, y)$. We have

$$
\left\{\begin{array}{l}
\phi_{t}+c \phi_{s}-e A(t, y) e \phi_{s s}+\nabla_{y} \cdot\left(e A(t, y) \phi_{s}\right)+e A(t, y) \nabla_{y} \phi_{s}-\nabla_{y} \cdot\left(A(t, y) \nabla_{y} \phi\right) \\
+q(t, y) \cdot \nabla_{y} \phi-e q(t, y) \phi_{s}-f(t, y, \phi)=0, \quad(t, s, y) \in \mathbb{R}^{2} \times \bar{\Omega}, \\
\nu A \nabla(\phi .1)) \text { on }(t, s, y) \in \mathbb{R}^{2} \times \partial \omega \\
\phi(t+T, s, y)=\phi(t, s, y) \quad \text { for all }(t, s, y) \in \mathbb{R}^{2} \times \bar{\omega}, \\
\lim _{s \rightarrow-\infty} \phi(t, s, y) \rightarrow 0, \lim _{s \rightarrow+\infty} \phi(t, s, y) \rightarrow p(t, y) \text { uniformly in }(t, y) \in \mathbb{R} \times \bar{\omega} .
\end{array}\right.
$$

In the present paper, we are interested in the existence of pulsating type entire solutions of (1.1), which are defined for all $(t, x, y) \in \mathbb{R} \times \bar{\Omega}$. The entire solutions have first been studied in the one dimensional case in various mathematical models, especially for equation (1.2) with monostable or bistable nonlinearities. See, for instance, Chen and Guo [7], Guo and Morita [14], Hamel and Nadirashvili $[\mathbf{1 5}, 16]$, Fukao, Morita, Ninomiya [8], Morita and Ninomiya [30] and Yagisita [45]. Recently, Li et al. [20] and Wang et al. [43] have investigated the entire solutions of the nonlocal diffusion equation with delayed nonlinearity. See also Li et al. [21] and Lv [22] for the Fisher-KPP equation with nonlocal dispersal and Wang et al. [42] for delayed lattice differential equations with monostable nonlinearity. Here, it needs to be pointed out that Chen and Guo [7] and Guo and Morita [14] developed an unified approach based on the comparison principle to find entire solutions for reaction-diffusion equations with both bistable and monostable nonlinearities. For high dimensional spaces, entire solutions has been obtained by Li et al. [23], Liu et al. [24] and Liu and Li [25] in infinite cylinders by considering a pair of traveling curved fronts. In $[\mathbf{2 3}], \mathrm{Li}$ et al. used the comparison argument to prove the existence of the entire solutions of reaction-advection- diffusion equations in infinite cylinders with both the monostable and the combustion nonlinearities. In [24], Liu et al. got the pulsating type entire solutions of reaction-advection-diffusion equations with monostable nonlinearity in space periodic framework by considering two pulsating traveling front connecting a constant unstable stationary state to a stable stationary state. Liu and Li $[\mathbf{2 5}]$ obtained the entire solutions for the bistable nonlinearity in heterogenous media by using a similar argument to Berestycki, Hamel and Matano [5] and Guo and Morita [14]. For other related results we refer to $[\mathbf{2 6 - 2 9 , 3 7 , 3 9 - 4 1 ]}$

From the dynamical points of view, the study of entire solution is essential for a full understanding of the transient dynamics and the structure of the global attractors. Also, entire solutions can be used to imply the dynamics of two solutions can have distinct histories in the configuration, though their asymptotic profiles as $t \rightarrow+\infty$ coincide, see Morita and Ninomiya [30]. It is well known that the existence, uniqueness and stability of the pulsating traveling fronts of the monostable reaction-advection-diffusion equations has been studied by Hamel [17] and Hamel 
and Roques [18]. However, the pulsating type entire solutions of (1.1) is still open, this motivate us to solve it.

Inspired by the construction in [14], we prove that, for any given direction vectors, there exist pulsating type entire solutions which behave as two pulsating fronts coming from both directions and approaching each other. Hereafter, for any given unit vectors pair $\left(e_{1}, e_{2}\right)$, where $e_{i}(i=1,2)$ satisfy $\left|e_{i}\right|=1(i=1,2)$ and $e_{2}=-e_{1}$, we consider a pair of pulsating fronts $\left(\phi_{i}, c_{i}\right)(i=1,2)$. Thus we can rewrite (1.10) as the following problem:

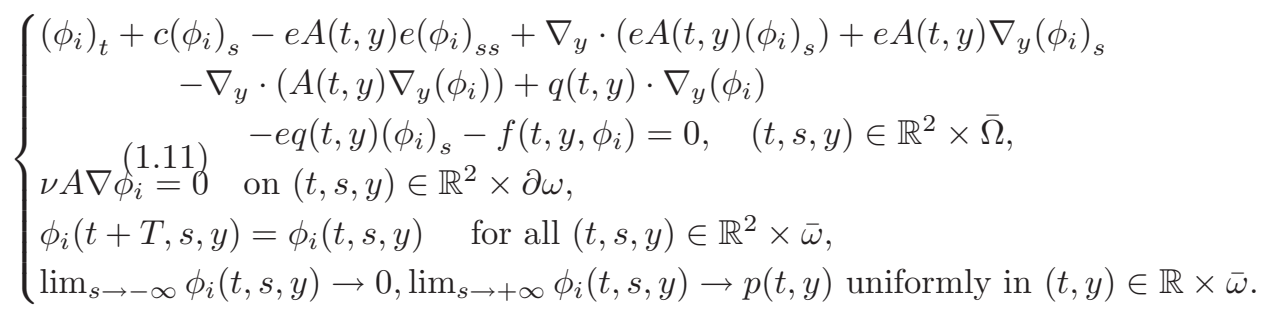

Our main result is as follows.

TheOREM 1.1. Assume that (1.5) and (1.9) hold. For any unit vectors pair $\left(e_{1}, e_{2}\right)$ with $e_{2}=-e_{1}$, let $\phi_{i}(t, s, y)(i=1,2)$ be the solutions of $(1.11)$ and $c^{*}\left(e_{i}\right)>$ $0(i=1,2)$ be the corresponding minimal pulsating traveling speeds. Then for any $c_{i} \geq c^{*}\left(e_{i}\right)$ and $\theta_{i} \in \mathbb{R}(i=1,2),(1.1)$ has an entire solution $u(t, x, y)$ that satisfies:

$$
\begin{aligned}
\lim _{t \rightarrow-\infty} & {\left[\sup _{x \cdot e_{1} \geq 0} \mid u(t, x, y)-\phi_{1}\left(t, c_{1} t-x \cdot e_{1}+\theta_{1}, y\right)\right.} \\
& \left.+\sup _{x \cdot e_{1} \leq 0}\left|u(t, x, y)-\phi_{2}\left(t, c_{2} t-x \cdot e_{2}+\theta_{2}, y\right)\right|\right]=0
\end{aligned}
$$

uniformly in $y \in \mathbb{R}^{\mathcal{N}-d}$. Moreover, we have

(i): $\frac{\partial u}{\partial t}>0,(t, x, y) \in \mathbb{R} \times \bar{\Omega}$;

(ii): $0<u<p(t, y),(t, x, y) \in \mathbb{R} \times \bar{\Omega}$;

(iii): $\lim _{t \rightarrow+\infty} u(t, x, y) \rightarrow p(t, y),(t, y) \in \mathbb{R} \times \bar{\omega}$.

This paper is organized as follows. In Section 2, we will give the exponential asymptotic behavior of the pulsating traveling fronts $\phi_{i}(t, s, y)(i=1,2)$ by considering the corresponding eigenvalue problem. IN Section 3 , we first deal with an ordinary differential system which plays an indispensable role in constructing the supersolution of (1.1), then we prove Theorem 1.1 by using the subsolution and supersolution constructed in (3.11) and (3.8). At last, we give some discussions in Section 4.

\section{Preliminaries}

In this section, we show the asymptotic behavior of the pulsating traveling fronts $\phi_{i}(t, s, y)(i=1,2)$.

Let $\zeta(t, y)$ be defined as in (1.6). For each $\lambda \in \mathbb{R}$, denote $k(\lambda)$ the principal eigenvalue of the following operator:

$$
\psi_{t}-\nabla \cdot(A \nabla \psi)+2 \lambda e A \nabla \psi+q \cdot \nabla \psi+\left[\lambda \nabla \cdot(A e)-\lambda q \cdot e-\lambda^{2} e A e-\zeta(t, y)\right] \psi=0
$$


with time periodicity conditions in $\mathbb{R} \times \bar{\omega}$ and boundary conditions $\nu A \nabla \psi=$ $\lambda(\nu A e) \psi$ on $\mathbb{R} \times \partial \omega$, and denote by $\psi_{\lambda}$ the unique positive principal eigenfunction such that $\|\psi\|_{L^{\infty}(\mathbb{R} \times \bar{\omega})}=1$. Suppose

$$
\mu_{0}<0,
$$

where $\mu_{0}<0$ denotes the principal eigenvalue of the linearized operator around 0 ,

$$
\psi(t, y) \mapsto \psi_{t}-\nabla \cdot(A(t, y) \nabla \psi)+q(t, y) \cdot \nabla \psi-\zeta(t, y) \psi,
$$

with time-periodicity conditions in $\mathbb{R} \times \bar{\omega}$ and Neumann boundary condition $\nu A \nabla \psi=$ 0 on $\mathbb{R} \times \partial \omega$ (with a slight abuse of notations, $\nabla \psi$ denotes $\left(0, \ldots, 0, \nabla_{y} \psi\right) \in$ $\left.\{0\}^{d} \times \mathbb{R}^{\mathcal{N}-d}\right)$. Define

$$
c^{*}(e)=\inf _{\lambda>0}\left(-\frac{k(\lambda)}{\lambda}\right),
$$

and for each $c>c^{*}(e)$, define $\lambda_{c}>0$ as

$$
\lambda_{c}=\min \left\{\lambda_{c}>0, k(\lambda)+c \lambda=0\right\} .
$$

These quantities are well-defined real numbers. Under all the assumptions above, (1.1) admits a pulsating traveling front when $c \geq c^{*}(e)$, and the pulsating traveling front is unique, monotone and stable. For more detailed results, we refer the reader to $[\mathbf{1 0}, \mathbf{1 7}, \mathbf{1 8}]$.

Utilizing the above result and inspiring by Hamel [17], we can obtain the exponential behavior of $\phi$ below in terms of the linear operator.

Lemma 2.1. ( [17]) Assume that (1.8)-(1.9) and (2.2) hold. Then there exists a constant $B>0$ such that

$$
\phi(t, s, y) \sim B e^{\lambda_{c} s} \psi_{\lambda_{c}}(t, y) \quad \text { as } s \rightarrow-\infty \quad \text { uniformly in }(t, y) \in \mathbb{R} \times \bar{\omega},
$$

if $c>c^{*}(e)$, and

$$
\phi(t, s, y) \sim B|s|^{2 m+1} e^{\lambda^{*} s} \psi_{\lambda^{*}}(t, y), \quad \text { as } s \rightarrow-\infty \quad \text { uniformly in }(t, y) \in \mathbb{R} \times \bar{\omega},
$$

if $c=c^{*}(e)$, where $\lambda^{*}>0$ is the unique root of the equation

$$
k(\lambda)+c^{*}(e) \lambda=0,
$$

and $2 m+1(m \in N)$ is the multiplicity of the solution $\lambda^{*}$.

From Lemma 2.1, we immediately have the following proposition.

Proposition 2.2. Under all the assumptions in Lemma 2.1, there exist some positive constants $k_{i}, K_{i}, \eta_{i}$ and $\lambda_{i}$ such that, for any $c_{i} \geq c^{*}\left(e_{i}\right)(i=1,2)$, $\phi_{i}(t, s, y)(i=1,2)$ satisfy

$$
\left\{\begin{array}{l}
k_{i} e^{\lambda_{i} s} \leq \phi_{i}(t, s, y) \leq K_{i} e^{\lambda_{i} s}, \quad \forall s \leq 0, \quad(t, y) \in \mathbb{R} \times \bar{\omega} \\
\partial_{s} \phi_{i}(t, s, y) \geq \eta_{i} \phi_{i}(t, s, y), \quad \forall s \leq 0, \quad(t, y) \in \mathbb{R} \times \bar{\omega},
\end{array}\right.
$$

where $i=1,2$. 


\section{Existence of the Pulsating Entire Solution}

In the present section, we prove our main result Theorem 1.1 by constructing appropriate subsolutions and supersolutions. Before giving the definition of the subsolutions and supersolutions, we first discuss the following ordinary differential equations which plays an important role in constructing supersolutions of (1.1):

$$
\left\{\begin{array}{l}
\dot{p}_{1}(t)=c_{1}+M e^{\alpha p_{1}(t)} \\
\dot{p}_{2}(t)=c_{2}+M e^{\alpha p_{1}(t)},
\end{array}\right.
$$

with the assumptions that $0<c_{1} \leq c_{2}, M>0$ and $\alpha>0$. Note that, $\dot{p}_{2}(t)-\dot{p}_{1}(t)=$ $c_{2}-c_{1} \geq 0$. By the additional assumption $p_{2}(0) \leq p_{1}(0)$, we obtain

$$
p_{2}(t)-p_{1}(t)-p_{2}(0)-p_{1}(0)=c_{2} t-c_{1} t \leq 0 \quad \text { for all } t \leq 0 .
$$

Thus $p_{2}(t) \leq p_{1}(t)(t \leq 0)$, if $p_{2}(0) \leq p_{1}(0)$.

Actually, we can solve the first equation of (3.1) explicitly as follows:

$$
p_{1}(t)=p_{1}(0)+c_{1} t-\frac{1}{\alpha} \log \left\{1+\frac{M}{c_{1}} e^{M p_{1}(0)}\left(1-e^{c_{1} \alpha t}\right)\right\} .
$$

Setting,

$$
\omega_{1}:=p_{1}(0)-\frac{1}{\alpha} \log \left\{1+\frac{M}{c_{1}} e^{M p_{1}(0)}\right\}
$$

and

$$
\omega_{2}:=\omega_{1}+p_{2}(0)+p_{1}(0) .
$$

Then we have

$$
p_{1}(t)-c_{1} t-\omega_{1}=-\frac{1}{\alpha} \log \left\{1-\frac{r}{1+r} e^{c_{1} \alpha t}\right\}, \quad r=\frac{M}{c_{1}} e^{\alpha p_{1}(0)} .
$$

It immediately follows that

$$
0<p_{1}(t)-c_{1} t-\omega_{1} \leq \mathcal{R}_{0} e^{c_{1} \alpha t} \quad \text { for all } t \leq 0,
$$

where $\mathcal{R}_{0}$ is some positive constant. Following a similar argument, we obtain the same estimate for $p_{2}(t)-c_{2} t-\omega_{2}$.

Next we give the explicit definition of the subsolutions and supersolutions of (1.1) on $(t, x, y) \in(-\infty, 0] \times \bar{\Omega}$

DeFinition 3.1. We call a function $\bar{u}(t, x, y) \in C^{1, \alpha / 2 ; 2, \alpha ; 2, \alpha}(t, x, y)$ is a supersolution of $(1.1)$ on $(t, x, y) \in(-\infty, 0] \times \bar{\Omega}$, if it satisfies:

$$
\left\{\begin{array}{l}
\bar{u}_{t}-\nabla \cdot(\nabla A(t, y) \bar{u}(t, x, y))+q(t, y) \cdot \nabla \bar{u}(t, x, y) \geq f(t, y, \bar{u}) \text { in }(t, x, y) \in \mathbb{R} \times \bar{\Omega} \\
\nu A \nabla \bar{u} \geq 0 \quad \text { on }(t, x, y) \in \mathbb{R} \times \partial \Omega
\end{array}\right.
$$

By reversing the inequality in (3.7), we can give the definition of the subsolution of (1.1) similarly.

Now, we start to construct a supersolution of (1.1).

Theorem 3.2. Assume that the nonlinearity $f$ satisfy (1.5) and (1.9). Let $\phi_{i}(t, s, y)$ be the solution of (1.11). Then for any unit vectors pair $\left(e_{1}, e_{2}\right)$ with $e_{2}=-e_{1}$ and $\left|e_{i}\right|=1,(i=1,2)$, and for $c_{i} \geq c^{*}\left(e_{i}\right)>0$, we can choose $\alpha$ and $M$ such that

$$
\bar{u}(t, x, y)=\phi_{1}\left(-x \cdot e_{1}+p_{1}(t), t, y\right)+\phi_{2}\left(-x \cdot e_{2}+p_{2}(t), t, y\right)
$$


is a supersolution of (1.1) for $t \leq 0$, where $\alpha$ and $M$ will be defined later.

Proof. Clearly, $\nu A \nabla \bar{u}=0$. Thus, it only remains to verify that

$$
\left.\mathcal{F}(\bar{u}):=\bar{u}_{t}-\nabla \cdot(\nabla A(t, y) \bar{u})+q(t, y) \cdot \nabla \bar{u}\right)-f(t, y, \bar{u}) \geq 0 \quad \text { for all } t \leq 0,
$$

which is equivalent to prove

$$
\begin{aligned}
\mathcal{F}(\bar{u})= & \sum_{i=1}^{2}\left[\phi_{i, t}+c \phi_{i, s}-e A(t, y) e \phi_{i, s s}+\nabla_{y} \cdot\left(e A(t, y) \phi_{i, s}\right)+e A(t, y) \nabla_{y} \phi_{i, s}\right. \\
& \left.-\nabla_{y} \cdot\left(A(t, y) \nabla_{y} \phi_{i}\right)+q(t, y) \cdot \nabla_{y} \phi_{i}-e q(t, y) \phi_{i, s}-f\left(t, y, \phi_{i}\right)\right] \\
= & \dot{p}_{1} \phi_{1, s}+\dot{p}_{2} \phi_{2, s}-c_{1} \phi_{1, s}-c_{2} \phi_{2, s}+f\left(t, y, \phi_{1}\right) \\
& +f\left(t, y, \phi_{2}\right)-f\left(t, y, \phi_{1}+\phi_{2}\right) \\
= & \phi_{1, s}\left(\dot{p}_{1}-c_{1}-\frac{G(t, x, y)}{\phi_{1, s}+\phi_{2, s}}\right)+\phi_{2, s}\left(\dot{p}_{2}-c_{2}-\frac{G(t, x, y)}{\phi_{1, s}+\phi_{2, s}}\right) \\
= & \left(\phi_{1, s}+\phi_{2, s}\right)\left(M e^{\alpha p_{1}}-U(t, x, y)\right),
\end{aligned}
$$

where

$$
\begin{gathered}
G(t, x, y)=f\left(t, y, \phi_{1}+\phi_{2}\right)-f\left(t, y, \phi_{1}\right)-f\left(t, y, \phi_{2}\right), \\
U(t, x, y)=\frac{G(t, x, y)}{\phi_{1, s}+\phi_{2, s}} .
\end{gathered}
$$

Define

$$
\lambda:=\min \left\{\lambda_{1}, \lambda_{2}\right\}
$$

Then we have $e^{\lambda_{2} p_{2}} \leq e^{\lambda p_{1}}$ and $e^{\lambda_{1} p_{1}} \leq e^{\lambda p_{1}}$ since $p_{2}(t) \leq p_{1}(t) \leq 0$. By the assumption (1.10) and $f(0)=0$, we have

$$
\begin{aligned}
G(t, x, y) & =f\left(t, y, \phi_{1}+\phi_{2}\right)-f\left(t, y, \phi_{1}\right)-f\left(t, y, \phi_{2}\right) \\
& \leq \phi_{2}\left[\int_{0}^{1} f_{u}\left(t, y, \phi_{1}+s \phi_{2}\right) d s-\int_{0}^{1} f_{u}\left(s \phi_{2}\right)\right] \\
& \leq \phi_{2}\left[\int_{0}^{1} f_{u}(t, y, 0) d s-\int_{0}^{1} f_{u}\left(s \phi_{2}\right)\right] \\
& \leq L \phi_{2}{ }^{2},
\end{aligned}
$$

where $L:=\max _{(t, y, u) \in \mathbb{R} \times \bar{\omega} \times[0,2]} \partial_{u u} f(t, y, u)$. It follows from a similar argument that

$$
G(t, x, y) \leq L \phi_{1}^{2} .
$$

In order to prove that $\mathcal{F}(\bar{u}) \geq 0$, we divide it to two cases.

A: $x \cdot e_{1} \geq 0$. Utilizing Proposition 2.2, we immediately have

$$
\begin{aligned}
U(t, x, y) & =\frac{G(t, x, y)}{\phi_{1, s}+\phi_{2, s}} \leq \frac{L \phi_{1}{ }^{2}}{\phi_{1, s}+\phi_{2, s}} \leq \frac{L \phi_{1}}{\eta_{1}+\phi_{2, s} / \phi_{1}} \\
& \leq \frac{L K_{1} e^{\lambda_{1}\left(-x \cdot e_{1}+p_{1}(t)\right)}}{\eta_{1}} \leq \frac{L K_{1}}{\eta_{1}} e^{\lambda_{1} p_{1}(t)} \\
& \leq \frac{L K_{1}}{\eta_{1}} e^{\lambda p_{1}(t)} .
\end{aligned}
$$


B: $x \cdot e_{1} \leq 0$, that is $x \cdot e_{2} \geq 0$. Similarly, we have

$$
\begin{aligned}
U(t, x, y) & \leq \frac{L \phi_{2}{ }^{2}}{\phi_{1, s}+\phi_{2, s}} \leq \frac{L \phi_{2}}{\eta_{2}+\phi_{1, s} / \phi_{2}} \\
& \leq \frac{L K_{2} e^{\lambda_{2}\left(-x \cdot e_{2}+p_{2}(t)\right)}}{\eta_{2}} \leq \frac{L K_{2}}{\eta_{2}} e^{\lambda_{2} p_{2}(t)} \\
& \leq \frac{L K_{2}}{\eta_{2}} e^{\lambda p_{1}(t)}
\end{aligned}
$$

Taking

$$
\alpha=\lambda, \quad M \geq \max \left\{\frac{L K_{1}}{\eta_{1}}, \frac{L K_{2}}{\eta_{2}}\right\} .
$$

Then we get $\mathcal{F}(\bar{u}) \geq 0$. Namely, $\bar{u}(t, x, y)=\phi_{1}\left(-x \cdot e_{1}+p_{1}(t), t, y\right)+\phi_{2}\left(-x \cdot e_{2}+\right.$ $\left.p_{2}(t), t, y\right)$ is a supersolution of (1.1). The proof is complete.

REMARK 3.3. In the proof of Theorem 3.2, we use the condition $c_{2} \geq c_{1}$. Indeed, by changing the variable $x \rightarrow-x$ and the invariance of (1.1), it is easy to show that Theorem 3.2 holds for $c_{2} \leq c_{1}$ since the function $p_{1}(t)$ and $p_{2}(t)$ exchange the roles.

Define

$$
\underline{u}(t, x, y):=\max \left\{\phi_{1}\left(c_{1} t-x \cdot e_{1}, t, y\right), \phi_{2}\left(c_{2} t-x \cdot e_{2}, t, y\right)\right\} .
$$

Clearly, $\underline{u}(t, x, y)$ is a subsolution of equation (1.1) in the sense of distribution since the maximum of subsolutions is also a subsolution.

Proof of TheOREM 1.1. Considering the following cauchy problem:

$$
\left\{\begin{array}{l}
u_{n, t}-\nabla \cdot\left(\nabla A(t, y) u_{n}\right)+q(t, y) \cdot \nabla u_{n}=f\left(t, y, u_{n}\right), \quad(t, x, y) \in[-n,+\infty) \times \bar{\Omega}, \\
\nu A \nabla u_{n}=0, \quad(t, x, y) \in(-n,+\infty) \times \partial \Omega, \\
u_{n, 0}:=u_{n}(-n, x, y)= \\
\max \left\{\phi_{1}\left(-x \cdot e_{1}-c_{1} n+\omega_{1},-n, y\right)+\phi_{2}\left(-x \cdot e_{2}-c_{2} n+\omega_{2},-n, y\right)\right\}, \quad(x, y) \in \Omega .
\end{array}\right.
$$

where $\omega_{1}$ and $\omega_{2}$ are defined by (3.3) and (3.4), respectively.

This problem is well posed and the maximum principle holds, since the domain $\omega$ is bounded and all the coefficients are periodic with respect to $t$. For a similar proof, we refer to Freedman [11].

First it follows from the maximum principle that

$$
u_{n}(t, x, y) \geq \underline{u}(t, x, y), \quad(t, x, y) \in[-n,+\infty) \times \Omega,
$$

where $u_{n}(t, x, y)$ is the unique classical solution of (3.12) that satisfies $0<u_{n}(t, x, y)<$ $p(t, y)$ for any $(t, x, y) \in[-n, 0] \times \Omega$ with $n>0(n \in \mathcal{N})$. On the other hand, Theorem 3.2 implies that

$u_{n}(t, x, y) \leq \phi_{1}\left(-x \cdot e_{1}+p_{1}(t), t, y\right)+\phi_{2}\left(-x \cdot e_{2}+p_{2}(t), t, y\right), \forall(t, x, y) \in[-n, 0] \times \Omega$.

By the standard parabolic estimates and passing $n \rightarrow+\infty$, we can obtain an entire solution $u(t, x, y)$ of (1.1) such that,

$$
\underline{u}(t, x, y) \leq u(t, x, y) \leq \bar{u}(t, x, y) .
$$


Note that, the entire solution obtained above is just a special case of Theorem 1.1 with $\theta_{1}=\omega_{1}$ and $\theta_{2}=\omega_{2}$. For any $\theta_{1}, \theta_{2} \in \mathbb{R}$, define

$$
\tilde{u}(t, x, y):=u(t-\tau, x+\xi, y) \text {, }
$$

where

$$
\xi:=\frac{c_{2}\left(\theta_{1}-\omega_{1}\right)-c_{1}\left(\theta_{2}-\omega_{2}\right)}{c_{1}+c_{2}} e_{2}
$$

and

$$
\tau:=\frac{\theta_{1}-\omega_{1}+\theta_{2}-\omega_{2}}{c_{1}+c_{2}} .
$$

Letting $\tilde{u}(t, x, y)$ by $u(t, x, y)$, then we find an entire solution of (1.1).

Next, we verify (1.11). Without loss of generality, we may assume that $c_{1} \leq c_{2}$.

We first consider the case $x \cdot e_{1} \geq 0$. It follows that

$$
\begin{aligned}
0 & <\phi_{1}\left(-x \cdot e_{1}+p_{1}(t), t, y\right)+\phi_{2}\left(-x \cdot e_{2}+p_{2}(t), t, y\right)-\phi_{2}\left(-x \cdot e_{2}+c_{2} t+\omega_{2}, t, y\right) \\
& \leq K_{1} e^{\lambda_{1} p_{1}(t)}+\sup _{s}\left|\phi_{2, s}\right| \mathcal{R}_{0} e^{c_{1} \lambda t} \\
& \leq K_{1} e^{\lambda p_{1}(t)}+\sup _{s}\left|\phi_{2, s}\right| \mathcal{R}_{0} e^{c_{1} \lambda t} \\
& \leq \mathcal{R}_{2} e^{c_{1} \lambda t}
\end{aligned}
$$

where $\mathcal{R}_{2}>0$ is some constant. Hence, we have

$$
\begin{aligned}
0 & \leq u(t, x, y)-\phi_{2}\left(-x \cdot e_{2}+c_{2} t+\omega_{2}, t, y\right) \\
& \leq \bar{u}(t, x, y)-p h i_{2}\left(-x \cdot e_{2}+c_{2} t+\omega_{2}, t, y\right) \\
& \leq \mathcal{R}_{2} e^{c_{1} \lambda t} .
\end{aligned}
$$

Similarly, when $x \cdot e_{1} \leq 0$, we have

$$
\begin{aligned}
& 0<\phi_{1}\left(-x \cdot e_{1}+p_{1}(t), t, y\right)+\phi_{2}\left(-x \cdot e_{2}+p_{2}(t), t, y\right) \\
& -\phi_{1}\left(-x \cdot e_{1}+c_{1} t+\omega_{1}, t, y\right), \leq \mathcal{R}_{1} e^{c_{1} \lambda t}
\end{aligned}
$$

and

$$
0 \leq u(t, x, y)-\phi_{1}\left(-x \cdot e_{1}+c_{1} t+\omega_{1}, t, y\right) \leq \mathcal{R}_{1} e^{c_{1} \lambda t}
$$

with $\mathcal{R}_{1}>0$ is some constant.

Taking $\mathcal{R}=\max \left\{\mathcal{R}_{1}, \mathcal{R}_{2}\right\}$, we obtain

$$
\begin{aligned}
0 \leq & \sup _{x \cdot e_{1} \leq 0}\left\{u(t, x, y)-\phi_{1}\left(-x \cdot e_{1}+c_{1} t+\omega_{1}, t, y\right)\right\} \\
& +\sup _{x \cdot e_{1} \geq 0}\left\{u(t, x, y)-\phi_{2}\left(-x \cdot e_{1}+c_{1} t+\omega_{2}, t, y\right)\right\} \\
\leq & \mathcal{R} e^{c \lambda t} .
\end{aligned}
$$

The proof is complete.

REMARK 3.4. If the nonlinearity $f$ in (1.1) is independent of $t$ and $y$, namely,

$$
f(t, y, u)=f(u)
$$

and $f$ satisfy

$$
\left\{\begin{array}{l}
f(0)=f(1)=0, f^{\prime}(1)<0 \\
f(s)>0, \quad \forall s \in(0,1) \\
f^{\prime}(s) \leq f^{\prime}(0) \quad \text { for all } s \in(0,+\infty)
\end{array}\right.
$$


By using the ordinary differential equation

$$
\zeta^{\prime}(t)=f(\zeta(t))
$$

we can prove that equation (1.1) with the nonlinearity $f$ satisfies (3.13) and (3.14) has another type of pulsating traveling entire solution $u_{i, k}(t, x, y)$ satisfying:

$$
\begin{aligned}
\max \left\{\chi_{i} \phi_{1}\left(-x \cdot e_{1}+c_{1} t+\theta_{1}, t, y\right), \quad\right. & \left.\chi_{k} \phi_{2}\left(-x \cdot e_{2}+c_{2} t+\theta_{2}, t, y\right), \zeta(t)\right\} \\
& \leq u_{i, k}(t, x, y) \leq \min \left\{\bar{u}_{i, k}(t, x, y), 1\right\},
\end{aligned}
$$

where

(3.16) $\bar{u}_{i, k}(t, x, y):=\chi_{i} \phi_{1}\left(-x \cdot e_{1}+p_{1}(t), t, y\right)+\chi_{k} \phi_{2}\left(-x \cdot e_{2}+p_{2}(t), t, y\right)+\rho(t)$ with $(i, k)=(1,0),(0,1),(1,1)$ are supersolutions for $t \in\left(-\infty,-T_{1}\right]$. For any $c_{i} \geq c^{*}\left(e_{i}\right)(i=1,2)$, and $\theta_{1}, \theta_{2} \in \mathbb{R}$, there exist monotone increasing functions $p_{j}(t)(j=1,2)$ satisfying

$$
\left|p_{j}(t)-c_{j} t-\theta_{j}\right| \leq \mathcal{R}_{1} e^{k_{j} t}
$$

where $T_{1}$ and $k_{j}$ are some positive constants, and the function $\rho$ sstisfies

$$
\rho(t)=\rho_{0} e^{\left(f^{\prime}(0) t\right)} \quad \text { and } \quad 0<\zeta(t)-\rho(t) \leq \mathcal{R}_{0} e^{\left(f^{\prime}(0) t\right)},
$$

where $\mathcal{R}_{0}$ is a positive constant.

\section{Discussion}

In this paper, we establish the existence of pulsating entire solutions of the reaction-advection-diffusion equation with a classical KPP nonlinearity by constructing appropriate subsolutions and supersolutions. Recently, Nadin [31] and Nolen et al. $[\mathbf{3 2}, \mathbf{3 3}]$ have obtained the existence of pulsating traveling fronts and theirs' qualitative properties in space-time periodic media, this motivate us to study pulsating entire solutions of the reaction-advection-diffusion equation in space-time periodic media, which we leave it for further studies.

\section{Acknowledgements}

The authors would like to thank the referees for their valuable comments and suggestions on the original manuscript.

\section{References}

[1] N. Alikakos, P. W. Bates, X. Chen, Traveling waves and locating oscillating patterns in multidimensional domains, Trans. Amer. Math. Soc. 351 (1999), 2777-2805.

[2] H. Berestycki and L. Nirenberg, Traveling fronts in cylinders, Ann. Inst. H. Poincaré Anal. Non Linéaire 9 (1992), 497-572.

[3] H. Berestycki, B. Larrouturou and P.L. Lions, Multi-dimensional travelling-wave solutions of a flame propagation model, Arch. Rational. Mech. Anl. 111 (1990), 33-49.

[4] H. Berestycki and F. Hamel, Generalized travelling waves for reaction-diffusion equations, in: Perspectivesin Nonlinear Partial Differential Equations: In Honor of Hïm Brezis, in: Contemp. MathSoc., Amer. Math. soc. 446 (2007), 101-123.

[5] H. Berestycki, F. Hamel and H. Matano, Bistable travelling waves around an obstacle, Comm. Pure Appl. Mth. 62 (2009), 729-788.

[6] X. Chen, Existence, uniqueness, and asymptotic stability of traveling waves in nonlocal evolution equations, Adv. Differential Equations 2 (1997), 125-160.

[7] X. Chen, J. S. Guo, Existence and uniqueness of entire solutions for a reaction-diffusion equation, J.Differential Equations 212 (2005), 62-84.

[8] Y. Fukao, Y. Morita and H. Ninomiya, Some entire solutions of the Allen-Cahn equation, Taiwanese J. Math. 8 (2004), 15-32. 
[9] R. A. Fisher, The advance of advantageous genes, Ann.Eugenics 7 (1937), 355-369.

[10] G. Fréjacques, Travelling waves in infinite cylinders with time-periodic coefficients, $\mathrm{PhD}$ thesis, 2006.

[11] A. Friedman, "Partial Differential Equations of Parabolic Type", Prentice-hall, INC. Englewood Cliffs, N.J. , 1964.

[12] M. Freidlin, On wave front propagation in periodic media, in: M. Pinsky (Ed.), Stochastic analysis and applications, Advances in Probability and Related Topics 7 (1984) 147-166.

[13] J. Gartner, M. Freidlin, The propagation of concentration waves in periodic and random media, Sov. Math. Dokl. 249 (1979), 521-525.

[14] J.S. Guo and Y. Morita, Entire solutions of reaction-diffusion equations and an application to discrete diffusive equations, Discrete Contin. Dyn. Syst. 12 (2005), 193-212.

[15] F. Hamel and N. Nadirashvili, Entire solutions of the KPP equation, Comm. Pure Appl. Math. 52 (1999), 1255-1276.

[16] F. Hamel and N. Nadirashvili, Traveling fronts and entire solutions of the Fisher-KPP equation in $\mathbb{R}^{N}$, Arch. Ration. Mech. Anal. 157 (2001), 91-163.

[17] F. Hamel, Qualitative properties of monostable pulsating fronts: Exponential decay and monotonicity, J. Math. Pures Appl. 89 (2008), 355-399.

[18] F. Hamel, L. Roques, Uniqueness and stability properties of monostable pulsating fronts, $J$. Eur. Math. Soc. 13 (2011), 345-390.

[19] A.N. Kolmogorov, I.G. Petrovsky, N.S. Piskunov, Etude de l'équation de la diffusion avec croissance de la quantitá de matière et son application àun problème biologique, BulletinUniversité d'Etat àMoscou Bull. Univ. Etat Moscou, Ser. Int. A 1 (1937), 1-25.

[20] W.T. Li, Z.C. Wang and J. Wu, Entire solutions in monostable reaction-diffusion equations with delayed nonlinearity, J.Differential Equations 245 (2008), 102-129.

[21] W.T. Li, Y.J. Sun and Z.C. Wang, Entire solutions in the Fisher-KPP equation with nonlocal dispersal, Nonlinear Anal. Real World Appl. 11 (2010), 2302-2313.

[22] G.Y. Lv, Asymptotic behavior of traveling fronts and entire solutions for nonlocal monostable equation, Nonlinear Anal. TMA 72 (2010), 3659-3668.

[23] W.T. Li, N.W. Liu and Z.C. Wang, Entire solutions in reaction-advection-diffusion equations in cylinders, J. Math. Pures Appl. 90 (2008), 492-504.

[24] N.W. Liu, W.T. Li and Z.C. Wang, Pulsating type entire solutions in monostable reactionadvection-diffusion equations in periodic excitable media, Nonlinear Anal. TMA, in press.

[25] N.W. Liu and W.T. Li, Entire solutions in bistable reaction-advection-diffusion equations in heterogeneous media, Science in china Series A: Mathrmatics 52 (2009), 1-15.

[26] G.Y. Lv, M.X. Wang, Existence, uniqueness and asymptotic behavior of traveling wave fronts for a vector disease model, Nonlinear Anal. Real World Appl. 11 (2010), 2035-2043.

[27] G.Y. Lv, M.X. Wang, Traveling wave front in diffusive and competitive Lotka-Volterra system with delays, Nonlinear Anal. Real World Appl. 11 (2010), 1323-1329.

[28] G.Y. Lv, M.X. Wang, Nonlinear stability of travelling wave fronts for delayed reaction diffusion equations, Nonlinearity 23 (2010), 845-873.

[29] G.Y. Lv, M.X. Wang, Existence, uniqueness and stability of traveling wave fronts of discrete quasi-linear equations with delay, Discrete Contin. Dyn. Syst. Ser. B 13 (2010), 415-433.

[30] Y. Morita and H. Ninomiya, Entire solutions with merging fronts to reaction-diffusion equations, J. Dynam. Differential Equations 18 (2006) 841-861.

[31] G. Nadin, Traveling fronts in space-time periodic media, J. Math. Pures Appl., 92 (2009), $232-262$.

[32] J. Nolen, M. Rudd, J. Xin, Existence of KPP fronts in spatially-temporally periodic advection and variational principle for propagation speeds, Dynamics of PDE 2 (2005), 1-24.

[33] J. Nolen, J. Xin, Existence of KPP type fronts in space-time periodic shear flows and a study of minimal speeds based on variational principle, Discrete Contin. Dynam. Systems 13 (2005), 1217-1234.

[34] J. Nolen, J. Xin, Reaction-diffusion front speeds in spatially-temporally periodic shear flows. SIAM J.Multiscale Model. Simul. 1 (2003), 554-570.

[35] N.Shigesada, K.Kawasaki, "Biological Invasions: Theory and Practice", Oxford Series in Ecology and Evolution, Oxford University Press, Oxford, 1997.

[36] N.Shigesada, K.Kawasaki, E.Teramoto, Traveling periodic waves in heterogeneous environments, Theor. Population Biol. 30 (1986), 143-160. 
[37] W.J. Sheng, N.W. Liu, Entire solutions in monostable reaction-advection-diffusion equations in infinite cylinders, Nonlinear Anal. TMA 74 (2011), 3540-3547.

[38] A. I. Volpert, V. A. Volpert and V. A. Volpert, "Traveling Wave Solutions of Parabolic Systems", Transl. Math. Monogr., vol. 140, Amer. Math. Soc. , Providence, 1994.

[39] Y.P. Wu, X.X. Xing, Q.X. Ye, Stability of travelling waves with algebraic decay for n-degree Fisher type equation, Discrete Contin. Dyn. Syst. 16 (2006), 47-66.

[40] Y.P. Wu, X.X. Xing, Stability of traveling waves with critical speeds for p-degree Fisher-type equations, Discrete Contin.Dyn. Syst. 20 (2008), 1123-1139.

[41] M.X. Wang, G.Y. Lv, Entire solutions of a diffusive and competitive Lotka-Volterra type system with nonlocal delays, Nonlinearity 23 (2010), 1609-1630.

[42] Z.C. Wang, W.T. Li, and J. Wu, Entire solutions in delayed lattice differential equations with monostable nonlinearity, SIAM J. Math. Anal. 40 (2009), 2392-2420.

[43] Z.C. Wang, W.T. Li and S. Ruan, Entire solutions in bistable reaction-diffusion equations with nonlocal delayed nonlinearity, Trans. Amer. Math. Soc. 361 (2009), 2047-2084.

[44] X. Xin, Existence and stability of traveling waves in periodic media governed by a bistable nonlinearity, J. Dynam. Differential Equations 3 (1991), 541-573.

[45] H. Yagisita, Backward global solutions characterizing annihilation dynamics of travelling fronts, Publ. Res. Inst. Math. Sci. 39 (2003), 117-164.

School of Mathematics and Statistics, Lanzhou University, Lanzhou, Gansu 730000 , PeOple's Republic of ChinA

E-mail address: shengwj09@lzu.edu.cn

School of Economics, Lanzhou University, Lanzhou, Gansu 730000, People's RepubLIC OF CHINA 